The experiments which have been described were made possible by the co-operation of members of the Cavendish High Voltage Laboratory, to whom I wish to express my thanks.
Cavendish Laboratory,
N. Feather.
Cambridge.

March 30.

1 Nature, 143, 330 (1939) ; Phys. Rev., 55, 418 (1939).

${ }^{2}$ Phys. Rev., 55, 417 (1939).

\section{An Effect of Gamma Radiation on Egg Albumin}

IT has previously been shown that the electrophoretic mobility of particles of inorganic sols and suspensions can be influenced by small doses of $\mathrm{X}$ and gamma-radiation. The mobility does not vary monotonically with the dose but exhibits a series of maxima and minima ${ }^{1,2}$.

A similar effect has now been observed with egg albumin. The technique employed was that of Freundlich and Abramson ${ }^{3}$, who have shown that the mobility of quartz particles covered with protein is equal to that of the protein itself. Samples of albumin in dilute acetate buffer solutions were exposed to increasing doses of gamma radiation, the irradiated solutions added to a quartz suspension, and the velocity of the composite particles under the influence of an electric field was determined with the ultramicroscope. Care was taken that sufficient protein was present to coat the quartz completely, and the buffer was maintained at the same strength in the initial and final solutions.

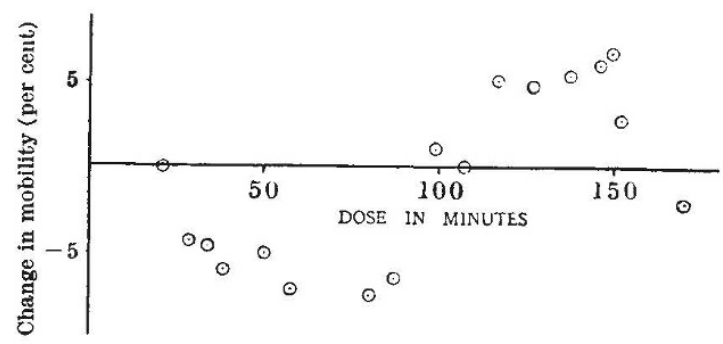

The results of a typical series of experiments are given in the accompanying graph, in which the percentage change of mobility is plotted against the time of exposure. A significant effect can already be observed after an exposure of 30 minutes, and as in the case of the inorganic sols, the curve relating the dose with the change of mobility passes through a minimum and a maximum. The probable error of a single velocity determination was found to be 0.5 per cent. The rate of irradiation as estimated from the amount and disposition of the radium employed was rather less than 3 röntgens per minute. Experiments are in progress to determine it with greater precision.

It is interesting to note that if unirradiated protein is added to a quartz suspension and the solution is then irradiated, the mobility of the composite particles remains unchanged over the range of doses investigated.

Department of Physics,

University of Reading.

J. A. Cro wTher. March 6.

${ }^{1}$ Crowther, J. A., and Liebmann, H., NATURE, 140, 28 (1937).

${ }^{2}$ Crowther, J. A., Liebmann, H., and Jones, R., Phil. Mag., 28, 120 (1938).

${ }^{3}$ Freundlich, H., and Abramson, H. A., Z. phys. Chem., 133, 51 (1928).

\section{A Positive Beam in Cathode Ray Tubes}

DURING the course of work on behalf of the Radio Research Board of the Department of Scientific and Industrial Research, certain phenomena have been observed in high vacuum cathode ray tubes of a commercial type.

The electrode system of this type of tube consists of an indirectly heated cathode, surrounded by a cylindrical electrode to control the electron quantity; an accelerating anode; a focusing anode; a final gun anode; and the two pairs of deflecting plates, each being connected separately. The fluorescent screen (blue, with short delay) is at a considerably lower potential than the gun anode, so that when electrons are projected from the gun with velocities corresponding to about eleven hundred volts or less, they slow up completely before reaching the screen, reverse their direction of travel, and finally return towards the gun. Under these latter conditions, when there is no fluorescent spot on the screen due to the electron beam, a faint, slightly more greenish spot has been observed, which can be brought to a comparatively sharp focus by reducing the potential on the electron focusing anode. Furthermore, the brilliance of this spot can be controlled by the electron quantity control electrode in the same manner as the electron beam.

This new spot first appears when the gun potential is about five hundred volts positive, increasing in brilliance up to a gun potential of about eleven hundred volts, above which it becomes unstable. With a further increase of potential the electron beam also reaches the screen, but also in a very unstable condition, wandering over the screen at random. During this condition, the newly observed spot is also present, in close proximity to the electron spot. Finally, when the potential is raised to about twelve hundred and fifty volts, the electron beam becomes stable and the new spot disappears. It is found that the screen has been badly damaged in the region of the spot, and it is thought that this damage may be due to bombardment by heavy particles moving relatively slowly.

Experiments made with electrostatic and magnetic deflecting fields indicate that the new spot observed under the above conditions is caused by a beam of positive ions. It has been further noticed that there is a very considerable external negative electrostatic charge on the glass of the tube in the region of the spot. This charge is sufficient to block the grid of an amplifying valve when a lead from the grid end of its high impedance input circuit is moved into contact with the glass in the region of the spot. Experiments made with an electrometer triode also show that there is a concentration of charge on the inner wall of the cathode ray tube having a positive potential of several hundred volts. It is a significant fact, when the nature of the beam is considered, that the electron beam produces no indication on the electrometer valve.

Considering the phenomena, especially the manner in which brilliance and focus are affected by the electron beam controls, it would appear that the production of positive ions is a secondary effect from the primary electron stream, possibly due to the slowing up of the beam and the slight trace of residual gas in the tube, although the tubes in question are of the hard or high vacuum type.

To sum up, it appears that positive ions can be generated in some types of cathode ray tubes having 